\title{
The relationship between burnout syndrome and low back pain, neck pain and mood status in hospital workers in the COVID-19 pandemic
}

\author{
İlknur Aykurt Karlıbel॰, Meliha Kasapoğlu Aksoy॰
}

Department of Physical Medicine and Rehabilitation, University of Health Sciences, Bursa Yüksek Ihtisas Training and Research Hospital, Bursa, Turkey

\begin{abstract}
Objectives: Burnout syndrome is expected among healthcare workers facing extended periods of physical and psychological stress. Physical and psychosocial factors affect the aetiology of healthcare workers' musculoskeletal pain. This study aimed to determine healthcare workers' burnout syndrome levels at a hospital during the COVID-19 pandemic, investigating burnout syndrome's relationship with lower back pain, neck pain, depression and anxiety.

Methods: This prospective observational study employed a Google Forms questionnaire, and 120 training and research hospital employees volunteered to participate. The questionnaire included the Maslach Burnout Inventory, Pain-Visual Analog Scale, Neck Disability Index, Oswestry Disability Questionnaire and Hospital Anxiety and Depression Scale.

Results: Fifty-four participants (45\%) had moderate emotional exhaustion, and 20 (16\%) had severe emotional exhaustion. Eighty-two (68\%) had mild depersonalisation and $38(32 \%)$ had moderate depersonalisation, and $120(100 \%)$ had severe reduced personal accomplishment. Moreover, $68.3 \%$ of volunteers complained of neck pain over the last year, while $51.7 \%$ complained of lower back pain. Relationships were observed between: emotional exhaustion, lower back pain, neck pain, lower back disability, neck disability, anxiety scores and depression scores; depersonalisation, neck pain, anxiety scores and depression scores; and personal accomplishment, neck pain, neck disability, back disability, anxiety scores and depression scores. Lower back disability was significantly higher among participants not infected with the SARS-CoV-2. Anxiety was significantly higher among participants infected with the SARS-CoV-2.

Conclusions: Burnout is a risk factor for musculoskeletal pain among healthcare workers. Therefore, healthcare workers require physical and psychological support during crises such as pandemics.

Keywords: Burnout syndrome; COVID-19; healthcare workers; lower back pain; neck pain; depression
\end{abstract}

Nince the World Health Organization (WHO) declared coronavirus disease 2019 (COVID-19) to be a pandemic, millions of cases and thousands of deaths have been confirmed [1]. For healthcare providers, working during an epidemic can have positive effects, improving post-traumatic growth through saving lives and improving patient outcomes [2]. However, due to COVID-19, a worldwide public 
health problem, healthcare workers also faced negative effects, such as the risk of transmission, inadequate medical equipment and devices, and particularly stressful workplace activity and intensity compared to the rest of society [3]. According to WHO data, more than 35,000 healthcare workers worldwide were infected with severe acute respiratory syndrome coronavirus 2 (SARS-CoV-2) during the pandemic, some of whom died as a result [1]. These difficulties and risks sparked fear, physiological stress and psychological stress among healthcare professionals [4]. Studies have reported that anxiety, depression, insomnia and high stress levels have been common among healthcare professionals during this pandemic [5-7]. These experiences can lead to post-traumatic stress disorder among healthcare workers. Post-traumatic stress disorder is expected to cause healthcare workers to feel burnt out after working under physical and psychological stress for long periods [2]. Burnout syndrome sufferers' mental state deteriorates alongside their work, family lives and social lives, negatively affecting their quality of life [8]. As a result, work-related tension and burnout can cause: psychological problems, such as chronic irritability, anxiety and depressive complaints; physical problems, such as fatigue, insomnia, stomach ailments, breathing difficulties and body aches; and decreased productivity and creativity with organisational consequences, such as workplace accidents [9]. Musculoskeletal disorders are a common occupational problem for healthcare professionals. In addition to physical factors - such as manual use, static tasks and repetitive tasks - psychosocial factors, such as work stress, monotonous tasks, high perceived workloads and time pressure, have been defined as work-related risk factors for musculoskeletal pain $[10,11]$. Back pain, lower back pain and neck pain are among healthcare workers' most common musculoskeletal problems [12]. Given this information, burnout levels caused by intense workloads and psychological stress may share a relationship with lower back and neck pain among healthcare workers during the COVID-19 pandemic.

Accordingly, this study aimed to determine healthcare workers' burnout syndrome levels at a hospital during the COVID-19 pandemic, investigating burnout syndrome's relationship with lower back pain, neck pain, depression and anxiety.

\section{METHODS}

This prospective observational study was planned in accordance with the Declaration of Helsinki and approved by our local ethics committee (2011-KAEK 25 2021/07-01). Participants' consent was obtained. The studied population comprised healthcare professionals aged 18-60 years who were working at a training and research hospital and who agreed to answer our questionnaire. Workers with less than one year of professional healthcare experience and workers who did not want to participate in our survey were excluded from the study. After providing necessary preliminary explanations and information to volunteers, we conducted a preliminary questionnaire with ten participants under observation. Subsequently, research data were collected online using an e-survey through Google Forms. In addition to questions about participants' socio-demographic information, the questionnaire used the following evaluation scales: the Maslach Burnout Inventory (MBI), the Visual Analogue Scale (VAS), the Oswestry Disability Questionnaire (ODQ), the Neck Disability Index (NDI) and the Hospital Anxiety and Depression Scale (HAD).

\section{Maslach Burnout Inventory (MBI)}

The MBI was developed to determine burnout levels. It comprises 22 items, and each item is scored between 0 and 6 . The composite score for each scale represents a participant's total score for each dimension. There are three components of this inventory: emotional exhaustion, depersonalisation and lack of personal accomplishment. Participants who score 0$16,17-26$ and $\geq 27$ on the emotional exhaustion scale exhibit low, moderate and high levels of emotional exhaustion, respectively. Those who score $0-6,7-12$ and $\geq 13$ on the depersonalisation dimension have low, moderate and high levels of depersonalisation, respectively. Items measuring lack of personal accomplishment are reverse-coded; participants with scores of 0-31, 32-38 and $\geq 39$ exhibit high, moderate and low lack of personal accomplishment, respectively [13].

\section{Visual Analogue Scale (VAS)}

Participants were asked to answer the following questions pertaining to the VAS: 'Did you have lower back pain last year?' and 'Did you have neck pain last year?' The VAS was used to evaluate the pain intensity 
of participants who reported experiencing lower back or neck pain. Patients were asked to rate their pain severity on a 10 -point scale ( 0 : I have no pain; 10 : very severe pain).

\section{Oswestry Disability Questionnaire (ODQ)}

The ODQ was used to assess disability due to lower back pain [14]. The questionnaire's validity and reliability were confirmed by Yakut et al. [15]. The ODQ comprises ten questions in total, each consisting of six options. The six options for each question are scored between 0 and 5 points. A score of $0-4$ is considered to indicate no disability, versus 5-14 indicating mild disability, 15-24 indicating moderate disability, 25-34 indicating severe disability and $>35$ indicating total disability.

\section{Neck Disability Index (NDI)}

The NDI is used to evaluate disability due to neck pain. Its validity and reliability were confirmed by Telci et al. [16]. Total NDI scores were used to determine participants' disability levels using the following assessment: 0-4 points: no disability; 5-14 points: mild disability; 15-24 points: moderate disability; 25-34 points severe disability; and $>35$ points: full disability.

\section{Hospital Anxiety and Depression Scale (HAD)}

The HAD is a self-assessment questionnaire comprising 14 questions. Seven questions (the odd-numbered items) measure anxiety (HAD-A), and the other seven (evenly numbered) measure depression (HADD). The HAD is a four-point Likert-type scale that is valid and reliable [17]. The HAD-A and HAD-D scores are calculated separately, yielding two scores that range between 0 and 21. High scores indicate high levels of anxiety or depression. Scores of 0-7 points indicate normal levels, 8-10 points indicate borderline abnormal levels and 11-21 points indicate abnormal levels.

Patients' burnout levels were calculated using three different components: emotional exhaustion, depersonalisation and lack of personal accomplishment. First, patients were divided into groups for participants with and without neck pain and with and without lower back pain. Then, they were further divided into two groups: participants exposed to SARS-CoV-2 (COVID-positive) and those not exposed to SARSCoV-2 (COVID-negative) (Fig. 1).

\section{Statistical Analysis}

Data were analysed using the IBM SPSS 23.0 statistics software package. Descriptive statistical methods (frequency, percentage, mean, standard deviation, median and min-max) were used to assess the data. Categorical variables were expressed as percentages n (\%). A chi-square test and Fisher's exact test were used to compare categorical data. The normal distribution of variables was calculated using the ShapiroWilk test. The data showed no normal distribution. A Mann-Whitney U test and Kruskal-wallis test were used for intergroup comparisons. The relationships between variables were assessed using Spearman's rho correlation test. The results were evaluated with a confidence interval of $95 \%$, and statistical significance was set at $p<0.05$.

\section{RESULTS}

This study's survey was sent to 130 healthcare professionals. Seven refused to answer the questionnaire. Three were excluded from the study because they had not completed one year of professional healthcare work experience (Fig. 1). A total of 120 professionals - including $36(30 \%)$ physicians, 44 (36.70\%) nurses, $24(20 \%)$ medical secretaries, eight (6.70\%) housekeepers and eight (6.70\%) administrative staff members - participated in this study. Ninetyfour $(78.30 \%)$ participants were women, and 86 (71.7\%) participants were over 30 years old. Participants' demographic and clinical data are shown in Table 1.

Fifty-four (45\%) participants had moderate burnout levels, and $20(16 \%)$ had severe levels of emotional exhaustion. Eighty-two (68\%) had mild levels of depersonalisation, and $38(32 \%)$ had moderate levels of depersonalisation. One hundred and twenty $(100 \%)$ had severe levels of reduced personal accomplishment. Participants' distribution by MBI score is presented in Table 1. Emotional burnout levels were statistically significantly higher among female participants than male participants and among participants working more than 40 hours per week compared to those working fewer than 40 hours per week ( $p<$ 0.05 ). Lack of personal accomplishment was higher among participants who were under 30 years old, female, single and working over 40 hours per week 


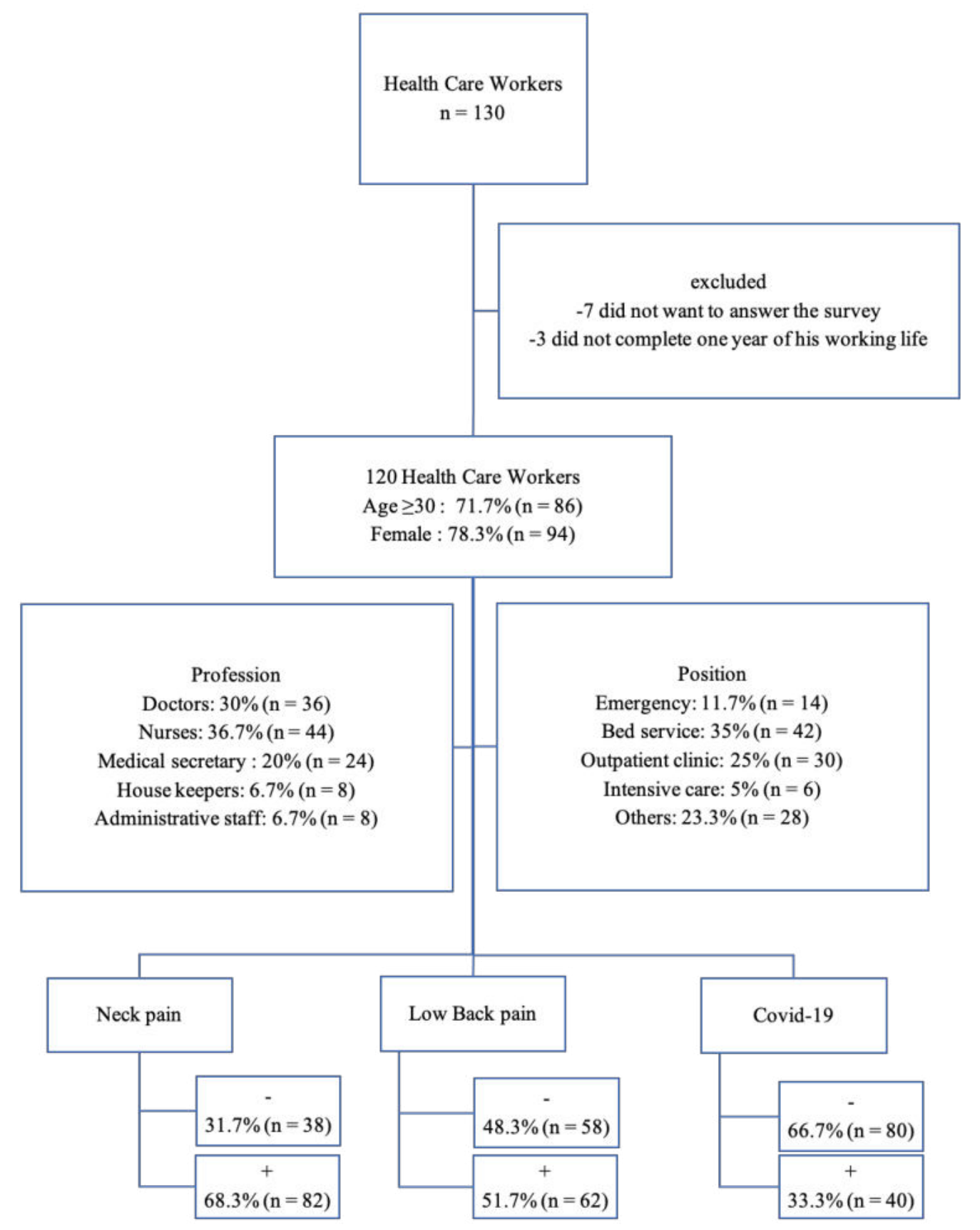

Fig. 1. Flow chart.

(Table 2).

Additionally, $68.3 \%$ of participants $(\mathrm{n}=82$; VAS $=2.86 \pm 2.70)$ complained of neck pain within the last year, and $51.7 \%(\mathrm{n}=62$; VAS $=4.25 \pm 2.18)$ complained of lower back pain within the last year (Fig. 1). When participants with and without neck pain were compared, no statistically significant difference in burnout levels was observed. When participants with and without lower back pain were compared in terms of burnout levels, only depersonalisation was statistically significantly higher among those with lower back pain (Table 3).

While a significant correlation was observed between neck pain (VAS) and all burnout scores, a significant correlation was found between lower back pain (VAS) and emotional burnout only. Both lower back and neck disability levels were found to correlate with emotional exhaustion and lack of personal accomplishment levels. A close relationship was also observed between emotional exhaustion, depersonalisation and lack of personal accomplishment levels and anxiety and depression scores (Table 
Table 1. Sociodemographic, clinical data and burnout levels of the participants

\begin{tabular}{|c|c|c|c|c|}
\hline Variables & & $\mathbf{n}$ & $\%$ & Mean \pm SD \\
\hline \multirow[t]{2}{*}{ Age (years) } & $<30$ & 34 & 28.3 & - \\
\hline & $\geq 30$ & 86 & 71.7 & - \\
\hline \multirow[t]{2}{*}{ Gender } & Female & 94 & 78.30 & - \\
\hline & Male & 26 & 21.70 & - \\
\hline \multirow[t]{2}{*}{ Marital status } & Married & 78 & 65 & - \\
\hline & Single & 42 & 35 & - \\
\hline \multirow[t]{3}{*}{ Education status } & High school/associate degree & 44 & 36.70 & - \\
\hline & Undergraduate & 44 & 36.70 & - \\
\hline & Postgraduate & 32 & 26.70 & - \\
\hline \multirow[t]{5}{*}{ Profession } & Doctor & 36 & 30 & - \\
\hline & Nurse & 44 & 36.70 & - \\
\hline & Medical secretary & 24 & 20 & - \\
\hline & Housekeepers & 8 & 6.70 & - \\
\hline & Administrative staff & 8 & 6.70 & - \\
\hline \multirow{5}{*}{ Position } & Emergency & 14 & 11.70 & - \\
\hline & Bed service & 42 & 35 & - \\
\hline & Outpatient clinic & 30 & 25 & - \\
\hline & Intensive care & 6 & 5 & - \\
\hline & Other & 28 & 23.30 & - \\
\hline \multirow[t]{2}{*}{ Weekly working hours } & $<40$ & 28 & 23.30 & - \\
\hline & $\geq 40$ & 92 & 76.70 & - \\
\hline \multirow[t]{3}{*}{ Way of working } & Daytime & 56 & 46.70 & - \\
\hline & Shift & 32 & 26.70 & - \\
\hline & 24 hours & 32 & 26.70 & - \\
\hline \multirow[t]{4}{*}{ Covid-19 } & I did not experience & 80 & 66.70 & - \\
\hline & I experienced it as a mild illness. & 32 & 26.70 & - \\
\hline & I experienced it as a severe illness. & 6 & 5 & - \\
\hline & I experienced without symptoms. & 2 & 1.70 & - \\
\hline \multirow[t]{3}{*}{ Neck pain } & None at all & 38 & 31.7 & - \\
\hline & Pandemic evolved during & 28 & 23.30 & - \\
\hline & Increased during the pandemic & 54 & 45 & - \\
\hline \multirow[t]{3}{*}{ Low back pain } & None at all & 58 & 48.30 & - \\
\hline & Pandemic evolved during & 36 & $30 \%$ & - \\
\hline & Increased during the pandemic & 26 & 21.70 & - \\
\hline \multicolumn{5}{|l|}{ Burnout levels } \\
\hline \multirow[t]{3}{*}{ Emotional exhaustion } & Mild 0-16 & 46 & 39 & $10.17 \pm 2.54$ \\
\hline & Moderate 17-26 & 54 & 45 & $20.74 \pm 2.38$ \\
\hline & Severe $\geq 27$ & 20 & 16 & $28.10 \pm 1.25$ \\
\hline \multirow[t]{3}{*}{ Depersonalization } & Mild 0-6 & 82 & 68 & $3.19 \pm 1.87$ \\
\hline & Moderate 7-12 & 38 & 32 & $8.15 \pm 1.05$ \\
\hline & Severe $\geq 13$ & 0 & 0 & - \\
\hline \multirow[t]{3}{*}{ Lack of personal accomplishment } & Severe $0-31$ & 120 & 100 & $20 \pm 3.69$ \\
\hline & Moderate $32-38$ & 0 & 0 & - \\
\hline & Mild $\geq 39$ & 0 & 0 & - \\
\hline NDI & & & & $11.18 \pm 7.47$ \\
\hline ODQ & & & & $7.28 \pm 6.59$ \\
\hline HAD-D & & & & $6.96 \pm 3.72$ \\
\hline HAD-A & & & & $9 \pm 3.96$ \\
\hline
\end{tabular}

NDI $=$ Neck Disability Index, ODQ = Oswestry Disability Questionnaire, HAD-D = The Hospital Anxiety and Depression Scale-Depression, HAD-A = The Hospital Anxiety and Depression Scale- Anxiety, Mean \pm SD; Rate \% 


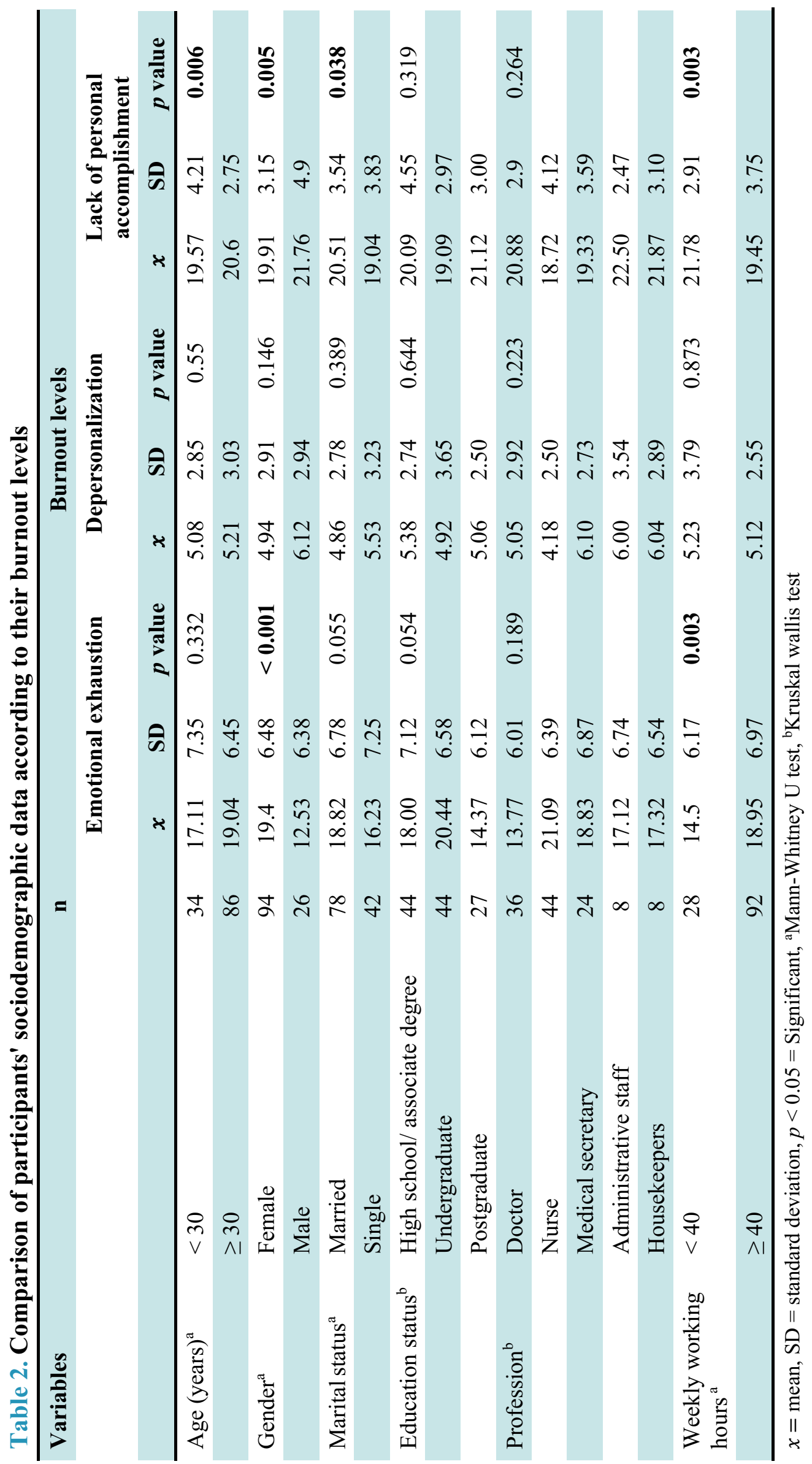


Table 3. Comparison of those with and without neck-low back pain in terms of burnout levels

n

\section{Burnout levels}

\section{Emotional exhaustion Depersonalization Lack of personal} accomplishment

\begin{tabular}{|c|c|c|c|c|c|c|c|c|c|c|c|}
\hline & & & $x$ & SD & p value & $x$ & SD & p value & $x$ & SD & p value \\
\hline \multirow[t]{2}{*}{ Neck pain } & + & 82 & 17.17 & 7.12 & 0.081 & 4.97 & 2.68 & 0.271 & 19.68 & 3.94 & 0.132 \\
\hline & - & 38 & 19.92 & 6.64 & & 4.31 & 3.17 & & 20.68 & 3.05 & \\
\hline \multirow[t]{2}{*}{ Low back pain } & + & 62 & 18.27 & 7.05 & 0.591 & 6.03 & 2.64 & $<0.001$ & 19.41 & 4.21 & 0.097 \\
\hline & - & 58 & 17.58 & 7.05 & & 3.58 & 2.51 & & 20.54 & 3.07 & \\
\hline
\end{tabular}

$x=$ mean, $\mathrm{SD}=$ standard deviation, $p<0.05=$ Significant, Mann-Whitney $\mathrm{U}$ test

Table 4. Relationships between burnout and neck-back pain (VAS), disability, depression, and anxiety in hospital workers

\begin{tabular}{|c|c|c|c|c|c|c|c|}
\hline & & \multicolumn{6}{|c|}{ Correlation } \\
\hline & & $\begin{array}{c}\text { Neck pain } \\
\text { VAS }\end{array}$ & NDI & $\begin{array}{c}\text { Low back pain } \\
\text { VAS }\end{array}$ & ODQ & HAD-D & HAD-A \\
\hline \multirow[t]{2}{*}{ Emotional exhaustion } & $\mathrm{R}$ & 0.447 & 0.541 & 0.280 & 0.553 & 0,678 & 0,642 \\
\hline & p value & $<0.001$ & $<0.001$ & 0.02 & $<0.001$ & $<0.001$ & $<0.001$ \\
\hline \multirow[t]{2}{*}{ Depersonalization } & $\mathrm{R}$ & 0.214 & 0.101 & -0.137 & 0,074 & 0,393 & 0,261 \\
\hline & p value & 0.019 & 0.338 & 0.136 & 0.485 & 0.002 & 0.042 \\
\hline \multirow{2}{*}{$\begin{array}{l}\text { Lack of personal } \\
\text { accomplishment }\end{array}$} & $\mathrm{R}$ & -0.194 & -0.294 & -0.28 & -0.428 & -0.506 & -0.433 \\
\hline & p value & 0.034 & 0.001 & 0.761 & $<0.001$ & $<0.001$ & $<0.001$ \\
\hline
\end{tabular}

NDI $=$ Neck Disability Index, ODQ = Oswestry Disability Questionnaire, HAD-D = The Hospital Anxiety and Depression Scale-Depression, HAD-A = The Hospital Anxiety and Depression Scale-Anxiety,

$p<0.05=$ Significant, Spearman's rho correlation test

Table 5. Comparison of burnout, depression, anxiety, neck and low back pain (VAS)disability levels between survivors of covid-19 disease and those who did not experience covid-19 disease.

\begin{tabular}{|c|c|c|c|}
\hline & COVID + & COVID - & $p$ value \\
\hline Emotional exhaustion & $17.50 \pm 7.60$ & $17.74 \pm 6.88$ & 0.656 \\
\hline Depersonalization & $4.30 \pm 3.92$ & $5.28 \pm 2.74$ & 0.127 \\
\hline Lack of personal accomplishment & $20.63 \pm 2.64$ & $19.85 \pm 3.83$ & 0.371 \\
\hline neck pain VAS & $2.66 \pm 2.62$ & $3.01 \pm 2.76$ & 0.485 \\
\hline NDI & $7.63 \pm 5.95$ & $12.94 \pm 8.03$ & 0.105 \\
\hline low back pain VAS & $4.45 \pm 1.94$ & $4.10 \pm 2.34$ & 0.375 \\
\hline ODQ & $5.10 \pm 5.84$ & $8.04 \pm 6,83$ & 0.008 \\
\hline HAD-D & $7.63 \pm 3.71$ & $6.13 \pm 3.66$ & 0.050 \\
\hline HAD-A & $10.16 \pm 4.64$ & $8.26 \pm 3.76$ & 0.048 \\
\hline
\end{tabular}

NDI = Neck Disability Index, ODQ = Oswestry Disability Questionnaire, HAD-D = The Hospital Anxiety and Depression Scale-Depression, HAD-A = The Hospital Anxiety and Depression Scale-Anxiety,

$p<0.05=$ Significant, Mann-Whitney $\mathrm{U}$ test 
4).

Forty participants $(33.3 \%)$ had previously had COVID-19. Eighty (66.70\%) had never had COVID19. Anxiety levels among participants who had had COVID-19 were statistically significantly higher than the corresponding levels among participants who had not had COVID-19. Lower back disability scores were statistically significantly higher among participants who had not had COVID-19 than among participants who had had COVID-19 (Table 5).

\section{DISCUSSION}

The results of this study showed that lower back and neck pain are common among healthcare workers experiencing the pandemic's physical and psychological burdens. Moreover, the groups who had and had not contracted COVID-19 were equally affected by lower back pain, neck pain and burnout syndrome scores. Sixteen per cent of participants exhibited high levels of emotional burnout, while all participants exhibited high levels of a lack of personal accomplishment. High levels of emotional burnout were common among women and participants working more than 40 hours per week, while a lack of personal accomplishment was common among participants who were under 30 years old, female, single and working over 40 hours per week. Positive correlations were observed between emotional exhaustion and lower back and neck pain, lower back and neck disability, anxiety scores and depression scores. A positive correlation was also observed between depersonalisation and neck pain, anxiety scores and depression scores. A negative correlation was observed between personal accomplishment and neck pain, neck disability and lower back disability, anxiety scores and depression scores. Additionally, participants who had not had COVID19 had higher lower back disability scores than participants who had contracted the disease. Anxiety scores were higher among participants who had had COVID-19 than among participants who had not.

Pandemics have physiological effects as well as psychological effects. Factors such as increased numbers of daily cases and rising death rates, inadequate medical equipment and devices, an active and intense work pace and stress can cause feelings of burnout among healthcare professionals [2, 3]. Giusti et al.
[18] reported that $67.6 \%$ of healthcare workers struggling with COVID-19 had moderate or severe emotional exhaustion, while $26 \%$ had moderate or severe depersonalisation and $74.4 \%$ experienced a moderate or severe decrease in personal accomplishment. In this study [18], predictors of all three components of burnout were work hours, psychological comorbidities, fear of infection and perceived support by friends. Additionally, the female gender, nursing, hospital work, and contact with COVID-19 patients were found to predict both emotional exhaustion and depersonalisation, while decreased personal accomplishment was estimated by age. In the current study, $61 \%$ of the participants had moderate or high levels of emotional exhaustion, while all participants had significant declines in personal accomplishment. Also, all participants exhibited mild or moderate depersonalisation. Factors such as duration of exposure to the pandemic's burdens, differences in working conditions and differences in the intensity of accompanying psychological comorbidities may explain these different results.

In the fight against COVID-19, nurses, women and frontline health workers have faced especially high psychological burdens [19]. Working life conditions contribute to burnout levels. Burnout is defined as a condition that mostly affects women [20]. In the COVID-19 pandemic, the results of a study of healthcare workers showed that gender has a major impact on emotional exhaustion and that women have higher levels of emotional exhaustion than men [21]. A study of Egyptian doctors found that older doctors were less likely to develop burnout during the COVID-19 pandemic. While the male gender predicted depersonalisation, the female gender was significantly associated with higher emotional exhaustion. Exposure to or death from COVID-19 among colleagues or relatives was significantly associated with high emotional exhaustion and decreased personal accomplishment, respectively. In this study, no relationship was observed between working hours and burnout [22]. In European countries, healthcare professionals - especially nurses - have been reported to exhibit high levels of stress, emotional fatigue and depressive symptoms [23]. A scoping study that included a total of 50 studies evaluated depression, anxiety, psychological trauma, sleep disorders, workplace burnout and fatigue among healthcare workers during the COVID-19 outbreak. It showed that the female gender, young elders, frontline 
workers and non-physician workers were more affected than other subgroups [24]. Factors such as nurses' working in close contact with infected patients, a lack of work experience among young people, a high number of women working in positions such as nurses or nursing assistants and a higher prevalence of mood disorders among women have been found to cause these results [25]. In the current study, in accordance with the literature [18, 20-22], participants' emotional exhaustion levels were statistically significantly higher among women than in men. Our result supported Giusti et al.'s [18] conclusion. However, contrary to a study by Abdelhafiz et al. [22], in the current study, emotional exhaustion levels were statistically significantly higher among participants working more than 40 hours per week compared to those working fewer than 40 hours per week. Though not to a statistically significant degree, nurses exhibited higher emotional burnout than other healthcare professionals. This result was also compatible with the literature [18, 22]. In addition, in line with the literature $[18,24]$, in the present study, we also observed a decrease in personal accomplishment among participants who were under 30 years old, female, single and working over 40 hours per week.

Musculoskeletal disorders are a common occupational problem for healthcare professionals. In addition to physical factors (such as manual use, static tasks and repetitive tasks), psychosocial factors (such as work stress, monotonous tasks, high perceived workloads and time pressure) have been defined as work-related risk factors for musculoskeletal diseases $[10,11]$. According to the results of a study investigating the prevalence of musculoskeletal pain among Norwegian nurses' assistants, neck, shoulder, elbow, high back and hip pain were more common among women than men. The prevalence of neck pain increased with increasing weekly work hours. Lower back pain was most common among people working in nursing homes [26]. In a study of 1,162 nurses in Japan, the prevalence of lower back pain during the previous 12 months was $71.3 \%$, versus $54.7 \%$ for neck pain and $33.9 \%$ for back pain. Neck pain was associated with tobacco smoking and high mental stress, while upper back and lower back pain were associated with bending or twisting, hard physical work, premenstrual tension, high mental pressure and insufficient staffing [27]. A study examining the relationship be- tween psychosocial work environments and musculoskeletal pain among nurses and nurses' assistants showed that lower back pain appeared to be associated with work fatigue. In contrast, symptoms in the neck and shoulders were mostly associated with relational and emotional factors [28]. The rates of neck pain and lower back pain in our study population were $68.3 \%$ and $51.7 \%$, respectively. When participants with and without neck pain were compared, we found no statistically significant difference in burnout component levels. However, correlations were observed between neck pain severity and emotional exhaustion, depersonalisation and a lack of personal accomplishment. When participants with and without lower back pain were compared in terms of burnout components, we found that only depersonalisation was statistically significantly higher among participants with lower back pain. Additionally, a significant relationship was found between the severity of lower back pain and emotional exhaustion. Moreover, we found a positive relationship between lower back and neck disability and emotional exhaustion, and we found a negative relationship between lower back and neck disability and a lack of personal achievement.

COVID-19 has been reported to cause musculoskeletal pain and sometimes last a long time [29]. The current study's results did not show significant differences in neck pain and lower back pain between participants who had contracted COVID-19 and participants who had not. Also, no significant difference was found in burnout or neck disability between participants who had contracted COVID-19 and participants who had not. Lower back disability was higher among participants who had not had COVID-19. Some participants who had contracted COVID-19 had been absent from their work environment for a while due to illness. However, others had continued to work without interruption. This difference may explain the higher incidence of lower back disability among participants who had not had COVID-19.

Iacovides et al. [30] found a significant relationship between depression and burnout in their study of 368 nurses. These researchers stated that there are two different types of burnout that are either related to depression or not related to depression. They argued that depression, which is associated with burnout, creates more severe burnout and develops among individuals who are prone to developing burnout [30]. During the 
COVID-19 pandemic, a study of Spanish medical personnel associated emotional exhaustion and depersonalisation with post-traumatic stress, while reduced personal accomplishment was associated with symptoms of anxiety and depression [25]. In the current study, we found a relationship between depression and anxiety scores and all burnout types. Additionally, we found that the anxiety scores of participants who had contracted COVID-19 were significantly higher than the corresponding scores of participants who had not.

\section{Limitations}

To the best of our knowledge, the present study is the first to investigate neck pain and lower back pain among healthcare workers professionally active during the COVID-19 pandemic and this pain's relationship with burnout, depression and anxiety that developed under the pandemic's burden. The limitations of our study are its relatively low number of participants and our lack of subgroup analysis. More comprehensive studies with subgroup analyses are needed.

\section{CONCLUSION}

During the COVID-19 pandemic, healthcare workers have had to work under more physical and psychological stress than normal. Our results reflected this situation: back and neck pain was common among healthcare personnel who were professionally active during the COVID-19 pandemic. Burnout levels were also high. The severity of lower back pain, neck pain and disability - as well as depression and anxiety may affect burnout levels among healthcare personnel experiencing heavy workloads during the pandemic. Emotional burnout levels were especially high among nurses who worked in close contact with infected people, female participants and participants working more than 40 hours per week. Participants who had contracted COVID-19 had worse lower back disability because they had worked longer than those who had not, and their anxiety levels were also higher. Given these results, burnout among healthcare workers can be considered a risk factor for musculoskeletal pain. For this reason, healthcare workers should be supported physically and psychologically during crises such as pandemics.

\section{Authors' Contribution}

Study Conception: IAK; Study Design: IAK; Supervision: İAK, MKA; Funding: İAK, MKA; Materials: İAK, MKA; Data Collection and/or Processing: İAK, MKA; Statistical Analysis and/or Data Interpretation: IAK, MKA; Literature Review: İAK; Manuscript Preparation: İAK and Critical Review: İAK, MKA.

\section{Conflict of interest}

The authors disclosed no conflict of interest during the preparation or publication of this manuscript.

\section{Financing}

The authors disclosed that they did not receive any grant during conduction or writing of this study.

\section{REFERENCES}

1. World Health Organization. Coronavirus disease (COVID2019) situation reports. 2020.

2. Chen R, Sun C, Chen JJ, Jen HJ, Kang XL, Kao CC, et al. A large-scale survey on trauma, burnout, and posttraumatic growth among nurses during the COVID-19 pandemic. Int J Ment Health Nurs 2021;30:102-16.

3. Arnetz JE, Goetz CM, Arnetz BB, Arble E. Nurse reports of stressful situations during the COVID-19 pandemic: qualitative analysis of survey responses. Int J Environ Res Public Health 2020;17:8126.

4. Spoorthy MS, Pratapa SK, Mahant S. Mental health problems faced by healthcare workers due to the COVID-19 pandemic: a review. Asian J Psychiatr 2020;51:102119.

5. Huang JZ, Han MF, Luo TD, Ren AK, Zhou XP. [Mental health survey of medical staff in a tertiary infectious disease hospital for COVID-19]. Zhonghua Lao Dong Wei Sheng Zhi Ye Bing Za Zhi. 2020; 38(3): 192-195. [Article in Chinese]

6. Lai J, Ma S, Wang Y, Cai Z, Hu J, Wei N, et al. Factors associated with mental health outcomes among health care workers exposed to coronavirus disease 2019. JAMA Netw Open 2020;3:e203976.

7. Lin K, Yang BX, Luo D, Liu Q, Ma S, Huang R, et al. The mental health effects of COVID-19 on health care providers in China. Am J Psychiatry 2020;177:635-6.

8. Maslach C, Schaufeli WB, Leiter MP. Job burnout. Annu Rev Psychol 2001;52:397-422.

9. Montero-Marin J, Prado-Abril J, Piva Demarzo MM, Gascon S, García-Campayo J. Coping with stress and types of burnout: explanatory power of different coping strategies. PLoS One 2014;9:e89090.

10. Richardson A, McNoe B, Derrett S, Harcombe H. Interventions to prevent and reduce the impact of musculoskeletal injuries among nurses: a systematic review. Int J Nurs Stud 2018;82:58- 
67.

11. Ahlberg-Hultén GK, Theorell T, Sigala F. Social support, job strain and musculoskeletal pain among female health care personnel. Scand J Work Environ Health 1995;21:435-9.

12. Richardson A, McNoe B, Derrett S, Harcombe H. Interventions to prevent and reduce the impact of musculoskeletal injuries among nurses: a systematic review. Int J Nurs Stud 2018;82:5867.

13. Slezáková Z, Vörösová G, Mičinová G. Burnout syndrome in neurological nursing. Clin Soc Work Health Interv 2016;7:3646.

14. Fairbank JC, Pynsent PB. The Oswestry Disability Index. Spine (Phila Pa 1976) 2000;25:2940-52; discussion 2952.

15. Yakut E, Düger T, Öksüz Ç, Yörükan S, Ureten K, Turan D, et al. Validation of the Turkish version of the Oswestry Disability Index for patients with low back pain. Spine 2004;29:581-5.

16. Telci EA, Karaduman A, Yakut Y, Aras B, Simsek IE, Yagli $\mathrm{N}$. The cultural adaptation, reliability, and validity of neck disability index in patients with neck pain: a Turkish version study. Spine (Phila Pa 1976);34;1732-5.

17. Snaith RP. The Hospital Anxiety And Depression Scale. Health Qual Life Outcomes 2003;1:29.

18. Giusti EM, Pedroli E, D'Aniello GE, Badiale CS, Pietrabisse G, Manna C, et al. The psychological impact of the COVID-19 outbreak on health professionals: a cross-sectional study. Front Psychol 2020;11:1684.

19. Lai J, Ma S, Wang Y, Cai Z, Hu J, Wei N, Wu J, et al. Factors associated with mental health outcomes among health care workers exposed to coronavirus disease 2019. JAMA Netw Open 2020;3:e203976.

20. Norlund S, Reuterwall C, Höög J, Lindahl B, Janlert U, Birgander LS. Burnout, working conditions and gender--results from the northern Sweden MONICA Study. BMC Public Health 2010;10:326.

21. Barello S, Palamenghi L, Graffigna G. Burnout and somatic symptoms among frontline healthcare professionals at the peak of the Italian COVID-19 pandemic. Psychiatry Res 2020;290:113129.

22. Abdelhafiz AS, Ali A, Ziady HH, Maaly AM, Alorabi M, Sultan EA. Prevalence, associated factors, and consequences of burnout among Egyptian physicians during COVID-19 pandemic. Front Public Health 2020;8:590190.

23. Zerbini G, Ebigbo A, Reicherts P, Kunz M, Messman H. Psychosocial burden of healthcare professionals in times of COVID19 - a survey conducted at the University Hospital Augsburg. Ger Med Sci 2020;18: Doc05.

24. Moitra M, Rahman M, Collins PY, Gohar F, Weaver M, Kinuthia J, Rössler W, et al. Mental health consequences for healthcare workers during the COVID-19 pandemic: a scoping review to draw lessons for LMICs. Front Psychiatry 2021;12:602614. 25. Luceño-Moreno L, Talavera-Velasco B, Garcia-Albuerne Y, Martin-Garcia J. Symptoms of posttraumatic stress, anxiety, depression, levels of resilience and burnout in Spanish health personnel during the COVID-19 pandemic. Int J Environ Res Public Health 2020;17:5514.

26. Eriksen W. The prevalence of musculoskeletal pain in Norwegian nurses' aides. Int Arch Occup Environ Health 2003;76:625-30.

27. Smith DR, Mihashi M, Adachi Y, Koga H, Ishitake T. A detailed analysis of musculoskeletal disorder risk factors among Japanese nurses. J Safety Res 2006;37:195-200.

28. Ahlberg-Hultén GK, Theorell T, Sigala F. Social support, job strain and musculoskeletal pain among female health care personnel. Scand J Work Environ Health 1995;21:435-9.

29. Disser NP, De Micheli AJ, Schonk MM, Konnaris MA, Piacentini AN, Edon DL, et al. Musculoskeletal consequences of COVID-19. J Bone Joint Surg Am 2020;102:1197-204.

30. Iacovides A, Fountoulakis KN, Moysidou C, Ierodiakonou C. Burnout in nursing staff: is there a relationship between depression and burnout? Int J Psychiatry Med 1999;29:421-33. 